\title{
Pseudocontinuous arterial spin labeling on magnetic resonance imaging in patients with carotid stenosis
}

\author{
SF Cheng, A Di Napoli, T Richards, MM Brown, HR Jäger \\ Division of Surgery and Interventional Science \& Institute of Neurology, University College London, United Kingdom \\ Neurosciences, Mental Health and Sensory Organs Department, Sapienza University, Rome, Italy
}

\section{Background}

- Arterial transit artefacts (ATA) are high signals on the surface of the brain, which is labeled blood that has not reached the capillary bed (gives an indication of brain perfusion).

- Aim: To determine the presence of ATAs on MRI in symptomatic and asymptomatic carotid stenosis and to evaluate its significance in these patients.

\section{Methods}

- 45 patients with internal carotid stenosis $\geqq 50 \%$ who had high resolution MRI perfusion imaging done were retrospectively included.

- Patients were divided into symptomatic and asymptomatic carotid stenosis, $50-70 \%$ and $\geq 70 \%$ stenosis.

- PcASL images were analysed and scored on the presence of ATA's in both hemispheres.

- The circle of Willis was scored on completeness (0: no Acom or Pcom; 1: Acom or Pcom; 2: both present).

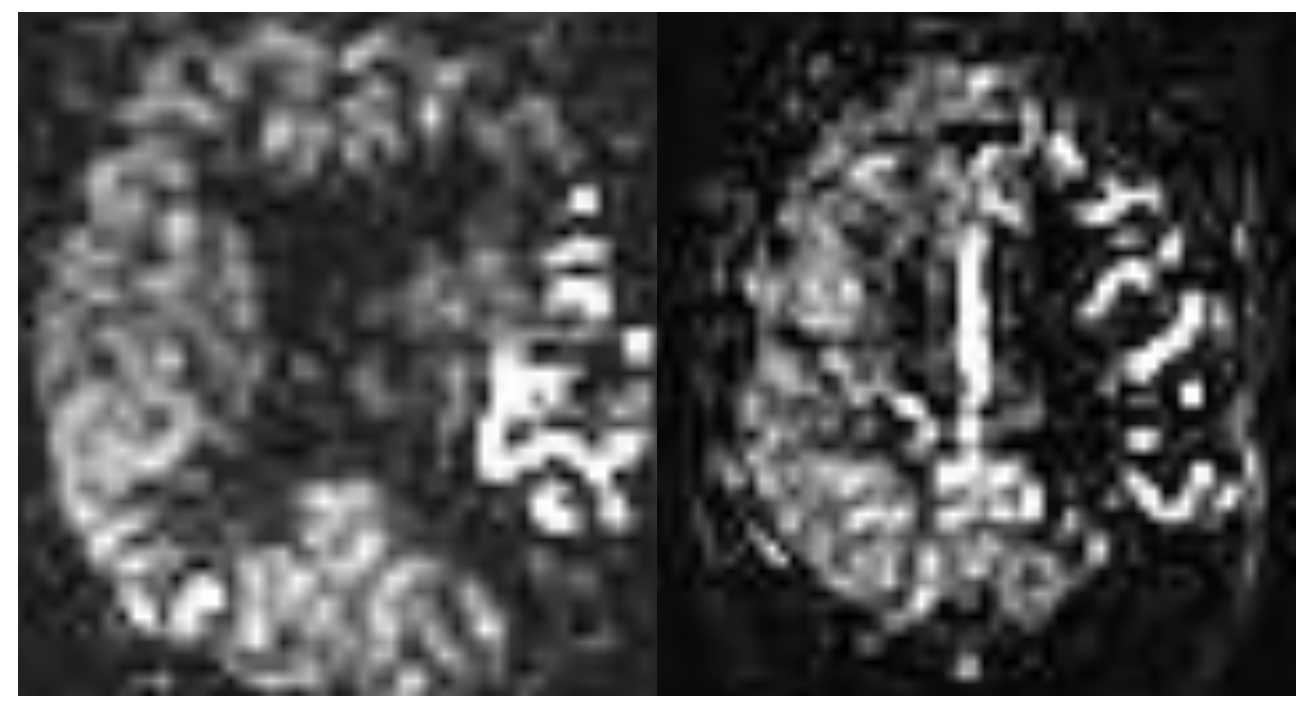

Figure 1. Pseudocontinuous arterial spin labelling on MRI.

\begin{tabular}{|c|c|c|c|c|}
\hline & & $\begin{array}{l}\text { ATA absent } \\
(n=29, \%)\end{array}$ & $\begin{array}{c}\text { ATA present } \\
(n=16, \%)\end{array}$ & $\begin{array}{c}\text { P- } \\
\text { value }\end{array}$ \\
\hline Age (years) & & $67.9 \pm 9.0$ & $76.2 \pm 8.0$ & 0.0038 \\
\hline Male gender & & $20(69)$ & $12 / 16(75)$ & 0.7432 \\
\hline $\begin{array}{l}\text { Ulcerated/irregular } \\
\text { plaque }\end{array}$ & & $9(31)$ & $8 / 16(50)$ & 0.3356 \\
\hline Stenosis $>70 \%$ & & $11(38)$ & $16 / 16(100)$ & $\begin{array}{c}<0.000 \\
1\end{array}$ \\
\hline $\begin{array}{l}\text { Intra plaque } \\
\text { haemorrhage }\end{array}$ & & $8(28)$ & $4 / 16(25)$ & 1 \\
\hline Circle of Willis & 0 & $2(7)$ & $9 / 16(56)$ & 0.0005 \\
\hline & 1 & $19(68)$ & $7 / 16(44)$ & \\
\hline & 2 & $7(25)$ & $0 / 16(0)$ & \\
\hline PCOM (contralateral) & & $6(21)$ & $3 / 16$ (19) & 1 \\
\hline PCOM (side of interest) & & $10(36)$ & $2 / 16(13)$ & 0.1601 \\
\hline ACOM & & $23(82)$ & $5 / 16(31)$ & 0.0012 \\
\hline Symptomatic & & $10(34 \%)$ & $13(81 \%)$ & 0.005 \\
\hline
\end{tabular}
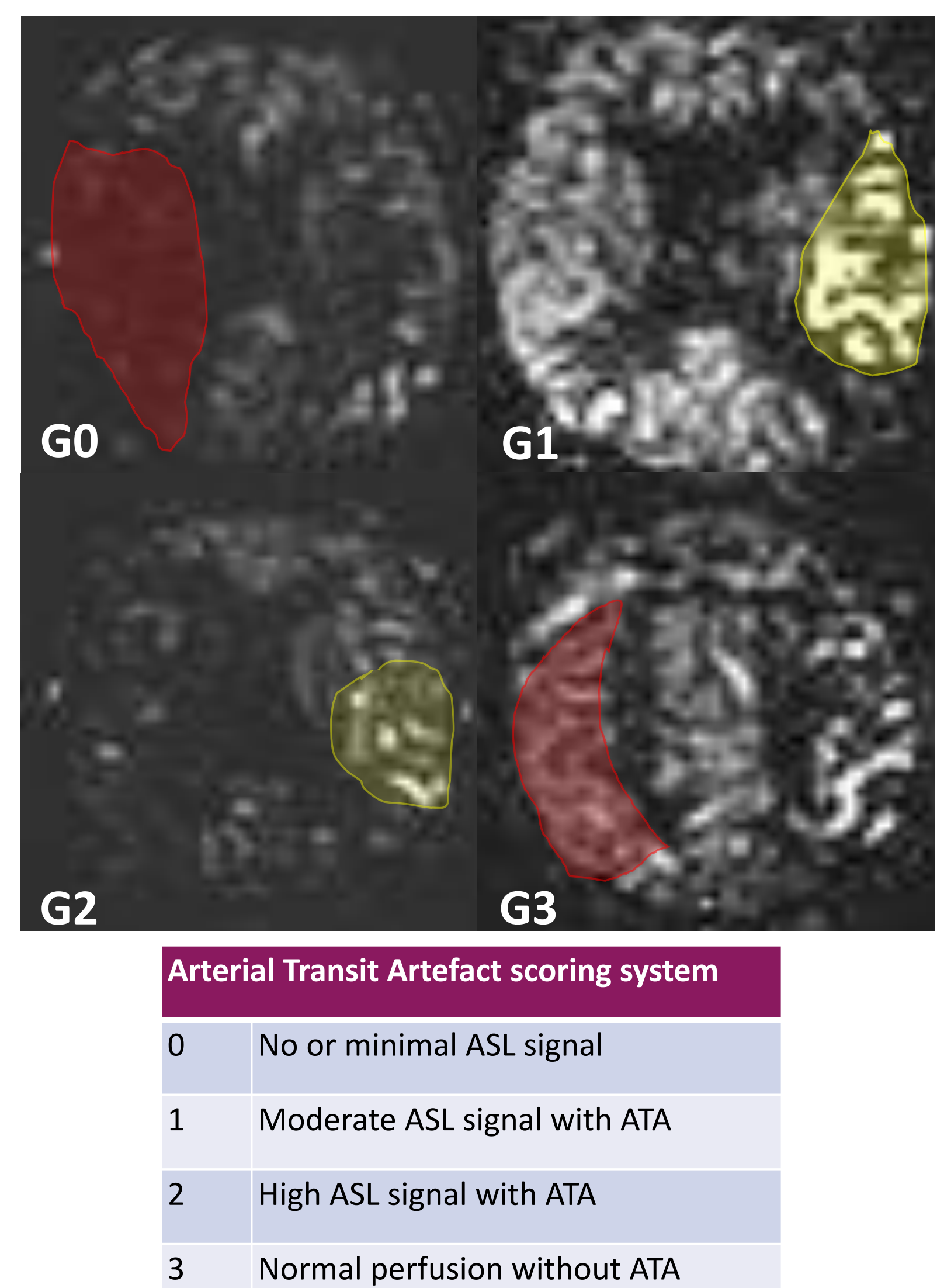

Figure 2. Arterial Transit Artefact Scoring System

\section{Results}

- Patients with $\geq 70 \%$ carotid stenosis all had ATAs $(n=16)$ compared to $38 \%$ of the patients with $50-70 \%$ stenosis $(n=11)$.

- Symptomatic patients were associated with the presence of ATAs $(81 \%$ in symptomatic vs. $34 \%$ asymptomatic; $\mathrm{p}=0.005)$

\section{Conclusion}

Only patients $\geqq 70 \%$ carotid stenosis were shown to have ATA's. Patients with symptomatic carotid stenosis more often had ATAs. 